\title{
Recurrent 8q13.2-13.3 microdeletions associated with Branchio-oto-renal syndrome are mediated by human endogenous retroviral (HERV) sequence blocks
}

\author{
Xiaoli Chen ${ }^{1 *+}$, Jun Wang ${ }^{2+}$, Elyse Mitchell ${ }^{3}$, Jin Guo ${ }^{1}$, Liwen Wang ${ }^{2}$, Yu Zhang ${ }^{1}$, Jennelle C Hodge ${ }^{3,4}$
} and Yiping Shen ${ }^{5,6,7^{*}}$

\begin{abstract}
Background: Human endogenous retroviral (HERV) sequences are the remnants of ancient retroviral infection and comprise approximately $8 \%$ of the human genome. The high abundance and interspersed nature of homologous HERV sequences make them ideal substrates for genomic rearrangements. A role for HERV sequences in mediating human disease-associated rearrangement has been reported but is likely currently underappreciated.

Methods and Results: In the present study, two independent de novo 8q13.2-13.3 microdeletion events were identified in patients with clinical features of Branchio-Oto-Renal (BOR) syndrome. Nucleotide-level mapping demonstrated the identical breakpoints, suggesting a recurrent microdeletion including multiple genes such as EYA1, SULF1, and SLCO5A1, which is mediated by HERV1 homologous sequences.
\end{abstract}

Conclusions: These findings raise the potential that HERV sequences may more commonly underlie recombination of dosage sensitive regions associated with recurrent syndromes.

Keywords: De novo 8q13.2-13.3 microdeletion, Human endogenous retroviral (HERV) sequences, Branchio-oto-renal syndrome, Mesomelia-synostoses syndrome

\section{Background}

Low-copy repeat (LCR) or segmental duplication (SD)mediated non-allelic homologous recombination (NAHR) is a common mechanism that results in microdeletions or microduplications [1]. Many of these rearrangements are responsible for human genomic disorders [2,3]. Typically SDs range from 10 to hundreds of kilobases with $>95 \%$ sequence identity and are involved in chromosomal recombination [4]; this usually occurs in a constrained footprint [5]. Indeed, smaller interspersed repeats like LINE, Alu, and human endogenous retrovirus (HERV) elements have also been shown to mediate recurrent genomic rearrangements

\footnotetext{
* Correspondence: cxlwx@sina.com; Yiping.Shen@childrens.harvard.edu ${ }^{\dagger}$ Equal contributors

'Beijing Municipal Key Laboratory of Child Development and Nutriomics, Capital Institute of Pediatrics, Beijing, China

${ }^{5}$ Shanghai Children's Medical Center, Shanghai Jiaotong University School of Medicine, Shanghai, China

Full list of author information is available at the end of the article
}

[5,6]. For example, whole genome analysis showed that $>16 \%$ of HERV-K elements have undergone rearrangements that result in larger-scale deletion/duplication and chromosome reshuffling in primate genomes [7]. In addition, homologous recombination between HERV15 elements on the $\mathrm{Y}$ chromosome mediates a recurrent $\mathrm{Y}$ chromosome microdeletion which removes the azoospermia factor a (AZFa) region that results in male infertility $[8,9]$. Finally, a recurrent translocation between 4q35.1 and $18 \mathrm{q} 22.3$ mediated by HERV-H recombination also has been reported recently [10].

Branchio-oto-renal syndrome (BOR, OMIM 113650) is an autosomal dominant disorder characterized by sensorineural, conductive, or mixed hearing loss, structural defects of the outer, middle, and inner ear, branchial fistulas or cysts, and renal abnormalities ranging from mild hypoplasia to complete absence [11]. The estimated prevalence of BOR syndrome is 1:40,000 and it affects 
approximately $2 \%$ of profoundly deaf children $[12,13]$. This syndrome is clinically and genetically heterogeneous and has a high penetrance with variable expressivity $[11,13]$. Mutations on the human ortholog of the Drosophila eyes absent gene (EYA1, OMIM 601653) are considered to be a major cause of BOR syndrome. Approximately 40\% of BOR patients have mutations in the EYA1 gene; 117 different heterozygous pathogenic variants including frame shift, stop, splice-site, and missense mutations have been reported [14-18]. Large heterozygous deletions at 8q13.213.3 encompassing the EYA1 gene have also been detected in patients with BOR [19-21]. Here we report two independent de novo 8q13.2-13.3 microdeletion events in two patients with clinical features of BOR. We provide evidence that this microdeletion is recurrent and mediated by HERV1 homologous sequences. Given that HERV sequences comprise approximately $8 \%$ of the human genome, our findings support the potential for these sequences to have a broader role in mediating recurrent disease-associated recombinations and polymorphic rearrangements on a genome-wide scale.

\section{Methods}

The Capital Institute of Pediatrics Review Board and the Mayo Clinic Institutional Review Board approved this project. The Written informed consent was obtained from the patient's guardian/parent/next of kin for the publication of this report and any accompanying images.

The physical and neurological examinations by a developmental specialist as well as biochemical and other medical evaluations were completed on case 1 at the Affiliated Children's Hospital of Capital Institute of Pediatrics. IQ was measured by Wechsler Intelligence Scale for Children (WISC). Phenotype information regarding case 2 was supplied by the Mayo Clinic Cytogenetics Laboratory at the time of clinical chromosomal microarray testing.

DNA from peripheral blood was isolated by the Blood and Tissue kit (Qiagen, Valencia, CA). Array CGH was performed using Agilent $244 \mathrm{~K}$ and $180 \mathrm{~K}$ oligonucleotide platform (Agilent Technologies Inc., Palo Alto, CA) for case 1 and 2, respectively.

Long-range PCR (Platinum PCR SuperMix High Fidelity kit, Invitrogen, Carlsbad, CA) was performed to locate the junction regions using multiple breakpoint-specific primers around the approximate breakpoints (Additional file 1: Table S1). The nested PCR was performed to confirm the deletion breakpoints in the two independent deletion cases. During the nested PCR, a $6.5 \mathrm{~kb}$ fragment outside two HERV blocks was first amplified from the long-range PCR products using the junction primers (1F and $1 \mathrm{R}$ in Additional file 1: Table S1) [22]. The $6.5 \mathrm{~kb}$ fragment was then purified from agarose gels using the QIAquick gel extraction kit (Qiagen, Valencia, CA) and diluted 1:10 to be used as the template for the second-round PCR (primers
2F and $2 \mathrm{R}$ in Additional file 1: Table S1). The junction fragment was, visualized on a $1 \%$ agarose gel, purified using Exonuclease I (New England Biolabs Inc., MA, USA), and then cloned into a TOPO-TA vector for Sanger sequencing following the manufacturer's protocol. A mixture of DNA from 10 normal children was used as the control.

\section{Results}

\section{Clinical reports}

Case 1 is a Chinese girl born to unrelated parents. Prenatal development was normal except for ultrasound detection of a cyst in the left kidney at 35 gestational weeks. She was born at 40 gestational weeks by Cesarean section with a birth weight of $4 \mathrm{~kg}$ and a birth length of $50 \mathrm{~cm}$. The Apgar scores were normal at both one and five minutes after birth. Abnormal hearing was noticed at newborn hearing screening. At three months of age, an initial CT scan diagnosed bilateral cochlear dysplasia with incompletely formed cochlea and posterior semicircular. At two years old, an auditory brainstem response (ABR) test confirmed moderate conductive hearing loss in both ears. On physical examination at four years of age, she was $110 \mathrm{~cm}$ (97th centile) and $16 \mathrm{~kg}$ (25-50th centile). The renal ultrasound showed bilateral hydronephrosis/pyelectasis. A routine urine test was negative and the levels of serum urea nitrogen $(3.1 \mathrm{umol} / \mathrm{L}$, normal range is 2.50 $8.21 \mathrm{mmol} / \mathrm{L}$ ) and creatinine $(53.2 \mathrm{umol} / \mathrm{L}$, normal range is 53-114.9umol/L) were consistent with normal renal function. Her second temporal CT scan performed at the age of two years showed bilateral inner ear malformations with cochlear hypoplasia (approximately 2 turns), incompletely formed lateral semicircular canal, enlargement of vestibular window and dilation of the right vestibular aqueduct. The images also manifested deep middle fossa. Her motor and neurological developmental skills were normal (IQ = 83) with the exception of language delay (vocabulary developmental index $=67$ ) due to hearing loss. The dysmorphic features included high arched palate, branchial anomalies and a preauricular pit, and a cupshape to the left low-set ear (Figure 1). The karyotype was normal 46, XX.

Case 2 is a two months old Middle Eastern male baby born to parents who are not documented to be related. Medical exam showed short stature, dysmorphic features including macrocephaly, malformed and low set ears, high arched palate, short neck, wide spaced eyes, and flat occiput. The karyotype was 46, XY. He was noted to have ear anomalies, although whether he has hearing loss is unknown based on the available information in his medical record.

\section{Array-CGH}

A 2.6 Mb microdeletion located at 8q13.2-13.3 was detected in both case 1 (chr8:70,062,624-72,714,795 in 


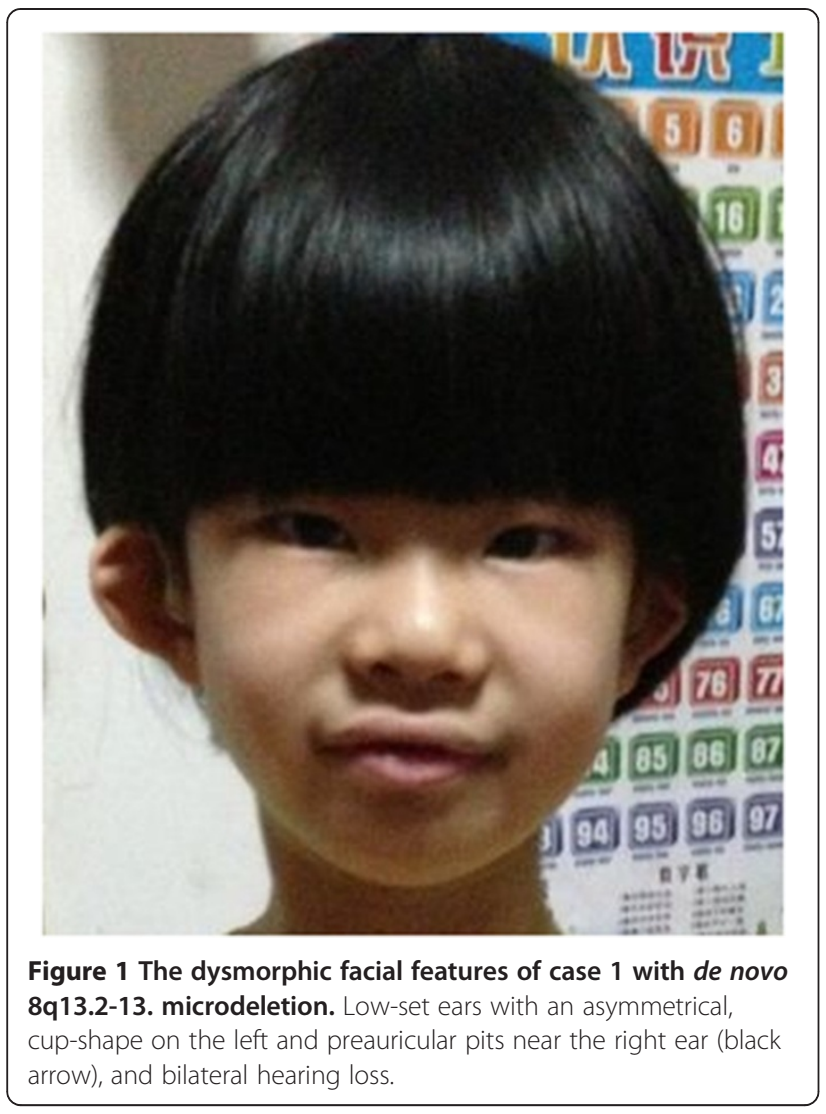

genome build hg18) and case 2 (chr8:70,062,624-72,738,255 in genome build hg18, Figure 2A). Parental testing confirmed they were both de novo deletions; array CGH and real-time quantitative PCR were employed in case 1 while FISH (BAC probe RP11-744 N15) was used for case 2. There are ten Refseq genes (LOC100505718, SULF1, SLCO5A1, PRDM14, NCOA2, TRAM1, LOC286190, $L A C T B 2, X K R$ 9, EYA1, Figure 2B) in this region and six genes reported in OMIM (SULF1, SLCO5A1, PRDM14, NCOA2, TRAM1, EYA1). In addition, one BOR patient reported by Sanchez-Valle et al. (chr8:70,053,688$72,748,049$ in genome build hg18) [22], and two cases in The International Standards for Cytogenomic Arrays Consortium (ISCA) dataset (http://www.iccg.org/; nssv578278 at chr8:69,400,250-72,388,412 and nssv584060 at chr8:70,062,670-72,714,817) had a similar size (Figure 2B). It is of note that another case in the ISCA dataset, ns1494921, is the same case as case 2 in our study. All five independent cases share similar breakpoints (labelled * in Figure 2B), suggesting the recurrent nature of this deletion.

\section{Breakpoint mapping}

The breakpoint-specific primer pair (BP-3 F and BP-3R in Additional file 1: Table S1) revealed an identical $8.5 \mathrm{~kb}$ amplicon in both case 1 and 2 which was not present in the control. To demonstrate the recurrent nature of the deletions and to understand their molecular mechanism, the junction region was mapped using longrange PCR and nested PCR design (The locations of the primers are shown in Figure 3A). Using the primer pair described in Sanchez-Valle et al. (1F and 1R in Additional file 1: Table S1, black dots in Figure 3A), an identical $6.5 \mathrm{~kb}$ junction fragment was also amplified in both cases (lane 3 and 4 in Figure 3B). This fragment is the same size as what was amplified in patient 1 in the report by Sanchez-Valle et al. [22]. Similarly, no amplification was detected for mixed control DNA. Thus, the junction sequences are deletion specific and two patients share similar breakpoints.

The nested PCR was performed ( $2 \mathrm{~F}$ and $2 \mathrm{R}$ in Additional file 1: Table S1, brown and blue dots in Figure 3A) using the $6.5 \mathrm{~kb}$ junction sequence as a template. An $800 \mathrm{bp}$ fragment was amplified from both cases (lane 11, 12 in Figure $3 \mathrm{~B}$ ). The sequences of the primer $2 \mathrm{~F}$ is not unique in the human genome, and so an 800 bp product was generated in the control as expected when genomic DNA was used as the template (lane 7-9 in Figure 3B). The sequence of the 800 bp product revealed that two human endogenous retroviral sequence blocks (HERVHint1, chr8:70,048,221-70,051,505 and HERVH-int2, chr8:72, $750,179-72,753,246$, identify is $>95 \%$, Figure $3 \mathrm{~A}$ ) are involved in this deletion. Using four locus-specific SNPs of the two HER regions (labeled black in Figure 4A, highlighted blue in Figure 4B) and two common SNPs (rs4737265 and rs28792781, red font in Figure 4B) as landmarks, the deletion breakpoints were narrowed to two 86 bp intervals (highlighted yellow in Figure 4B) which are identical between the two adjacent HERV blocks (chr8:70,050,79870,050,884 and chr8:72,752,537-72,752,623).

\section{Discussion}

Human Endogenous Retroviral (HERV) sequences, which are remnants of past retroviral infection, make up approximately $8 \%$ of the human genome [23]. The interspersed nature and high sequence homology of HERVs make them ideal substrates for NAHR in the human genome [24]. In fact, greater than $16 \%$ of human HERV-K elements may have mediated large-scale genome rearrangements during primate evolution [7], and inverted HERV-K elements are known to cause the frequent 8p23.1 polymorphic inversions in humans [25]. It is known that homologous recombination between distant HERVs can cause inherited male infertility due to loss of the 792-kb fragment encompassing azoospermia factor a (AZFa) region [8,9]. Sanchez-Valle et al. reported the first BOR patient with 8q13.2-13.3deletion and proposed that this microdeletion was mediated by two adjacent HERV blocks [22]. A genome-wide copy number variant analysis was performed for a Branchiooto-renal syndrome cohort with negative pathogenic 


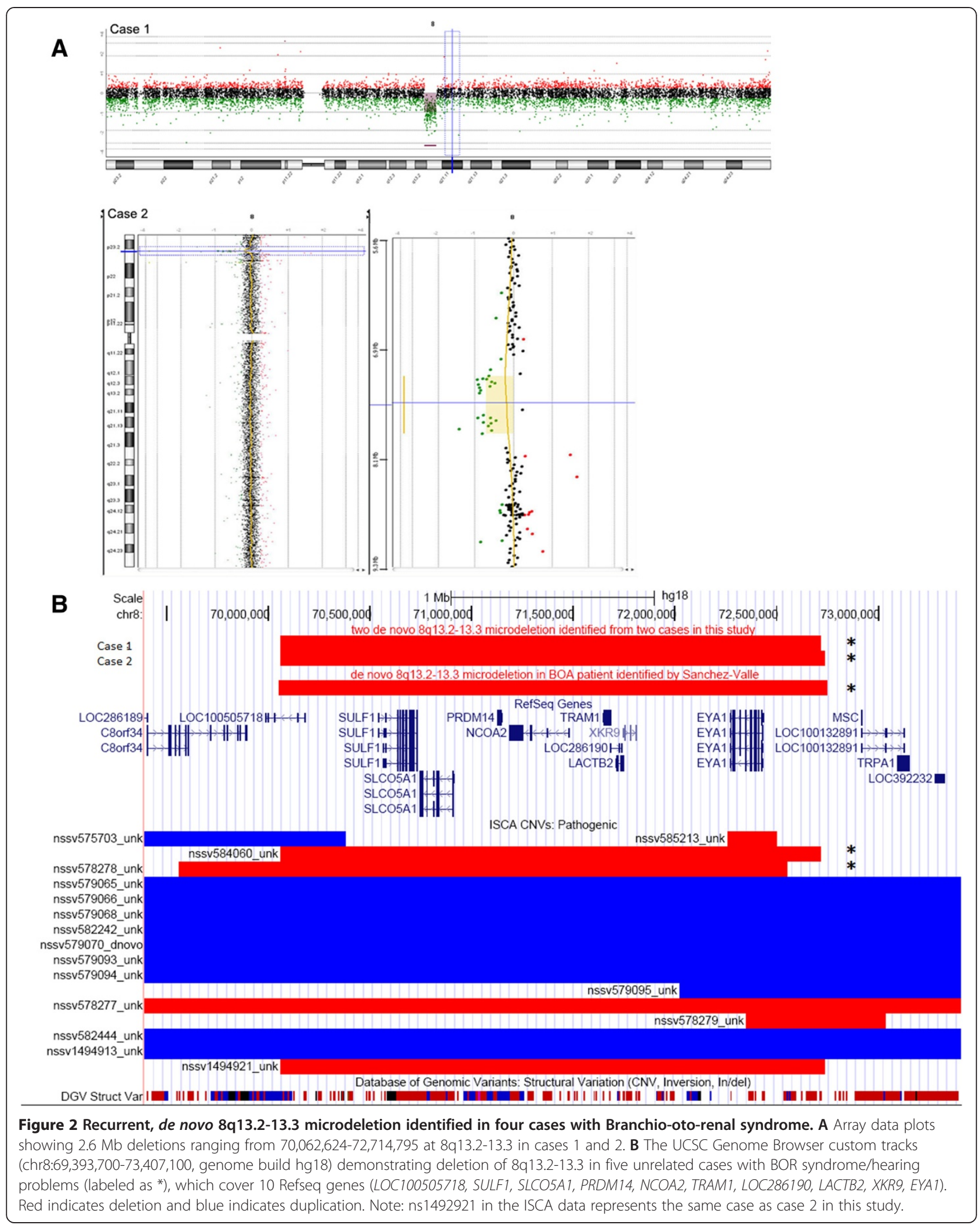




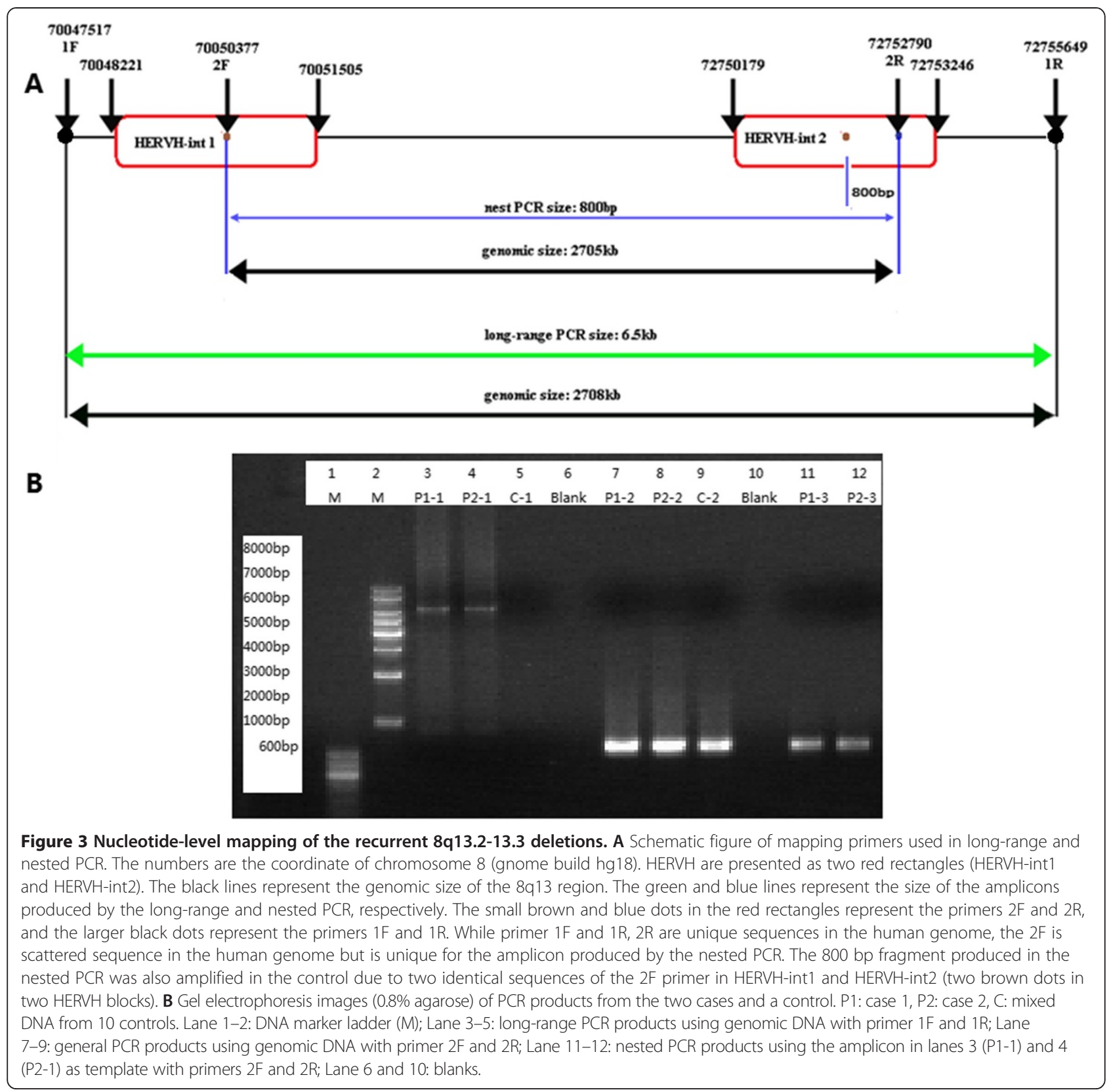

mutation in known BOR-causing genes [26]. Four carriers with 8q13.2-13.3 deletions (2.7 Mb deletion, chr8:70053668-72748108) were identified that share approximatly identical breakpoints covering HERV blocks, proving 8q13.2-13.3 deletion is a recurrent genomic rearrangement event.

In the current study, we mapped the breakpoint of 8q13.2-13.3 deletions in two unique cases, and found the breaks occurred in identical 86 bp homologous sequence within HERV regions. We therefore demonstrated that 8q13.2-13.3 deletions associated with BOR syndrome features can be recurrent through an NAHR mechanism, which places this microdeletion syndrome into the same category as DiGeorge syndrome and William's syndrome. Similarly, HERV sequences were reported to mediate recurrent 3q13.2-q13.31 deletions which cause a new syndrome of hypotonia and motor, language, and cognitive delays [27]. Beside such inter-chromosomal arrangements, $150 \mathrm{bp}$ homologous sequences of two HERV-H blocks have been shown to mediate a recurrent intra-chromosomal translocation between 4q and 18q [10]. The above published studies and our results suggest that HERV regions can induce both inter-chromosomal and intra-chromosomal rearrangements.

8q13.2-13.3 microdeletion has been associated with several medical disorders due to haploinsufficiency of 


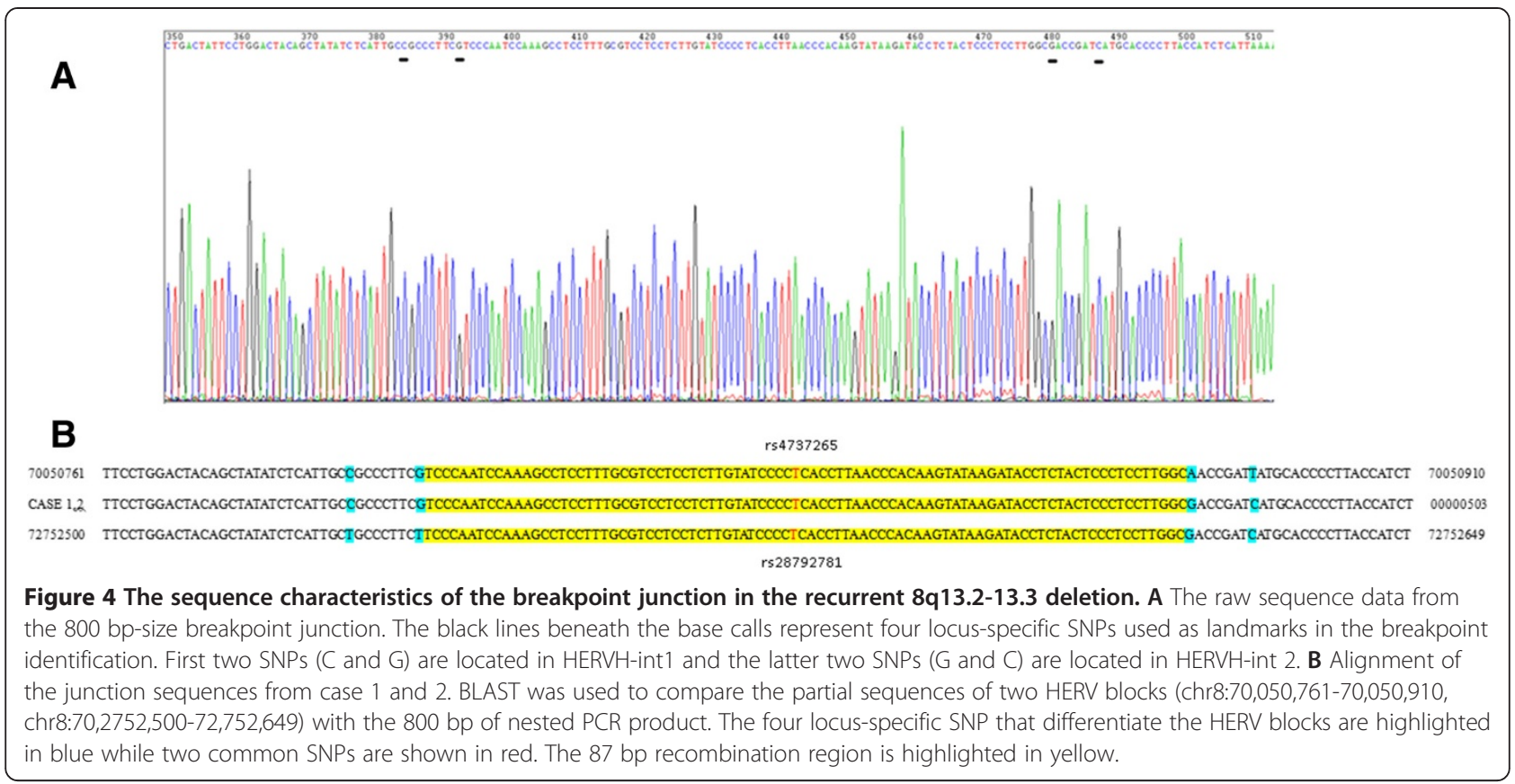

genes within the region. Deletion of EYA1 is associated with BOR syndrome $[11,19,20,28]$. The typical manifestations of BOR are hearing loss and structural defects of the outer, middle, and inner ear (98.5\%), preauricular pits (83.6\%), branchial anomalies (68.5\%), renal anomalies (38.2\%), and external ear abnormalities (31.5\%) [11]. 8q13 has also reportedly been associated with Mesomelia-Synostoses syndrome (MSS, OMIM 600383) due to the co-deletion of SULF1 and SLCO5A1 [29]. MSS is a rare dominantly inherited disorder characterized by mesomelic limb shortening and acral synostoses, renal malformations and/or congenital heart defects occur sporadically. Hearing loss has not been reported in MSS patients [30,31], although $<10$ patients with MSS have been described [29,32]. We summarized the clinical phenotypes of reported carriers with 8q13.2-13.3 microdeletion carriers reported in the literatures (Table 1) [22,26]. Neither mesomelic short stature nor severe skeletal changes was observed. Their predominant clinical features include hearing loss $(4 / 6)$, branchial fistulae (5/6), preauricular pits (4/6) and

Table 1 Clinical findings in six patients with recurrent 8q13.2-13.3 deletion

\begin{tabular}{|c|c|c|c|c|c|c|c|}
\hline & & $\begin{array}{l}\text { Case } 1 \text { in } \\
\text { this study }\end{array}$ & $\begin{array}{l}\text { Case } 2 \text { in this } \\
\text { study }\end{array}$ & Patient $1 *$ & $21230 * *$ & $20960 * *$ & $518240^{* *}$ \\
\hline \multirow[t]{4}{*}{ Major BOR criteria } & Hearing loss/impairment & + & NA & + & - & + & + \\
\hline & Branchial fistulae & + & NA & + & + & + & + \\
\hline & Preauricular pits & + & NA & + & + & - & + \\
\hline & $\begin{array}{l}\text { Hydronephrosis/kidney } \\
\text { agenesis }\end{array}$ & + & NA & + & + & - & + \\
\hline Minor BOR criteria & $\begin{array}{l}\text { Ear anomalies or ear } \\
\text { symptoms (cup ear, } \\
\text { low-set ear, asymmetry ear) }\end{array}$ & + & + & + & + & + & - \\
\hline $\begin{array}{l}\text { Dysmorphic facial } \\
\text { features }\end{array}$ & & $\begin{array}{l}\text { High arched } \\
\text { palate }\end{array}$ & $\begin{array}{l}\text { Macrocephaly, high } \\
\text { arched palate, short } \\
\text { neck, wide spaced eyes, } \\
\text { and flat occiput }\end{array}$ & $\begin{array}{l}\text { Left-sided } \\
\text { microphthalmos } \\
\text { with iris coloboma }\end{array}$ & $\begin{array}{l}\text { Small mouth, } \\
\text { branchial tag }\end{array}$ & - & - \\
\hline \multirow[t]{3}{*}{$\begin{array}{l}\text { Non-BOR related } \\
\text { abnormalities }\end{array}$} & $\begin{array}{l}\text { Intellectual disability/ } \\
\text { developmental delay }\end{array}$ & - & NA & + & - & - & - \\
\hline & Speech/language delay & + & NA & + & - & - & - \\
\hline & Short stature & - & $\begin{array}{l}\text { Proportional short } \\
\text { stature }\end{array}$ & $\begin{array}{l}\text { Proportional short } \\
\text { stature }\end{array}$ & - & - & - \\
\hline
\end{tabular}


hydronephrosis/kidney agenesis (4/6), which are major suggestive phenotypes of BOR syndrome. Our literature review suggests that recurrent 8q13.2-13.3 deletion is an important cause of BOR. However, not all BOR cases result from recurrent 8q13.2-13.3 deletion by a HERV mediated non-allelic homologous recombination mechanism. Some BOA patients have non-recurrent alternations, such as the EYA1 exonic deletion, exemplified by case nssv525813 in the ISCA dataset (Figure 2B). Based on published diagnostic scoring criteria for BOR [16], case 1 in this study and four patients in the literatures meet the definition of typical BOR (hearing loss and preauricular pits, inner ear and renal anomalies), while case 2 in this study represents atypical BOR (ear anomalies or ear symptoms). Thus the results are consistent with phenotype heterogeneity of the 8q13.2-13.3 deletion. The functional polymorphism of the EYA1 hemizygous allele or another dosage sensitive gene in the deleted region could potentially be responsible for the phenotype heterogeneity.

\section{Conclusion}

In summary, we confirmed in two cases the recurrent nature of two 8q13.2-13.3 microdeletion associated with BOR syndrome. We also mapped the deletion breakpoints and demonstrated that the deletion is mediated by two adjacent HERVH sequences. Therefore, this microdeletion represents another example of HERVmediated NAHR, indicating a more prominent role for the HERV regions in recurrent genomic disorders.

\section{Additional file}

Additional file 1: Table S1. Primers used for long-range PCR and nested PCR.

\section{Competing interests}

The authors declare that they have no competing interests.

\section{Authors' contributions}

$\mathrm{XLC}$ and YPS designed the study, performed the data analysis and manuscript writing. XLC contributed to long-range and nested PCR; JW and LWW helped in patient recruitment and clinical checkup and obtaining a genetics questionnaire; JG performed DNA extraction and an array experiment; $Y Z$ performed the clone and gel purification and sequencing; $\mathrm{EM}$ and $\mathrm{JH}$ helped in patient recruitment and contributed to manuscript writing. All authors read and approved the final manuscript.

\section{Acknowledgments \\ We appreciate the patients and their families for their participation. This work was supported by the National Natural Science Foundation of China (No. 81371903 to YPS and 81100841 to XLC), Beijing Municipal Science \& Technology Commission (Z131107002213159 to JW), The capital health research and development of special to XLC, and the Chinese Returned Oversea Scientist Fund to XLC from Beijing Science and Technology.}

\section{Author details}

${ }^{1}$ Beijing Municipal Key Laboratory of Child Development and Nutriomics, Capital Institute of Pediatrics, Beijing, China. ${ }^{2}$ Department of Neurology, Affiliated Children's Hospital of Capital Institute of Pediatrics, Beijing, China.
${ }^{3}$ Department of Laboratory Medicine and Pathology, Mayo Clinic, Rochester, MN, USA. ${ }^{4}$ Department of Pathology and Laboratory Medicine, Cedars-Sinai Medical Center, Los Angeles, CA, USA. ${ }^{5}$ Shanghai Children's Medical Center, Shanghai Jiaotong University School of Medicine, Shanghai, China.

${ }^{6}$ Department of Pathology, Harvard Medical School, Boston, MA, USA. 'Department of Laboratory Medicine, Children's Hospital Boston, Boston, MA, USA.

Received: 3 September 2013 Accepted: 18 July 2014

Published: 19 August 2014

\section{References}

1. Bailey JA, Eichler EE: Primate segmental duplications: crucibles of evolution, diversity and disease. Nat Rev Genet 2006, 7:552-564.

2. Stankiewicz P, Lupski JR: Genome architecture, rearrangements and genomic disorders. Trends Genet 2002, 18:74-82.

3. Lupski JR: Genomic disorders: structural features of the genome can lead to DNA rearrangements and human disease traits. Trends Genet 1998, 14:417-422.

4. Rudd MK, Keene J, Bunke B, Kaminsky EB, Adam MP, Mulle JG, Ledbetter $\mathrm{DH}$, Martin CL: Segmental duplications mediate novel, clinically relevant chromosome rearrangements. Hum Mol Genet 2009, 18:2957-2962.

5. Batzer MA, Deininger PL: Alu repeats and human genomic diversity. Nat Rev Genet 2002, 3:370-379.

6. Deininger PL, Moran JV, Batzer MA, Kazazian HH Jr: Mobile elements and mammalian genome evolution. Curr Opin Genet Dev 2003, 13:651-658.

7. Hughes JF, Coffin JM: Evidence for genomic rearrangements mediated by human endogenous retroviruses during primate evolution. Nat Genet 2001, 29:487-489.

8. Blanco P, Shlumukova M, Sargent CA, Jobling MA, Affara N, Hurles ME: Divergent outcomes of intrachromosomal recombination on the human $Y$ chromosome: male infertility and recurrent polymorphism. J Med Genet 2000, 37:752-758

9. Sun C, Skaletsky H, Rozen S, Gromoll J, Nieschlag E, Oates R, Page DC: Deletion of azoospermia factor a (AZFa) region of human $\mathrm{Y}$ chromosome caused by recombination between HERV15 proviruses. Hum Mol Genet 2000, 9:2291-2296.

10. Hermetz KE, Surti U, Cody JD, Rudd MK: A recurrent translocation is mediated by homologous recombination between HERV-H elements. Mol Cytogenet 2012, 5:6.

11. Kochhar A, Fischer SM, Kimberling WJ, Smith RJ: Branchio-oto-renal syndrome. Am J Med Genet A 2007, 143A:1671-1678.

12. Fraser FC, Sproule JR, Halal F: Frequency of the branchio-oto-renal (BOR) syndrome in children with profound hearing loss. Am J Med Genet 1980, 7:341-349.

13. Lindau TA, Cardoso ACV, Rossi NF, G CM: Anatomical changes and audiological profile in Branchio-oto-renal syndrome: a literature review. Int Arch Otorhinolaryngol 2014, 18:68-76.

14. Estefania E, Ramirez-Camacho R, Gomar M, Trinidad A, Arellano B, Garcia-Berrocal JR, Verdaguer JM, Vilches C: Point mutation of an EYA1-gene splice site in a patient with oto-facio-cervical syndrome. Ann Hum Genet 2006, 70:140-144.

15. Abdelhak S, Kalatzis V, Heilig R, Compain S, Samson D, Vincent C, Weil D, Cruaud C, Sahly I, Leibovici M, Bitner-Glindzicz M, Francis M, Lacombe D, Vigneron J, Charachon R, Boven K, Bedbeder P, Van Regemorter N, Weissenbach J, Petit C: A human homologue of the Drosophila eyes absent gene underlies branchio-oto-renal (BOR) syndrome and identifies a novel gene family. Nat Genet 1997, 15:157-164.

16. Chang EH, Menezes M, Meyer NC, Cucci RA, Vervoort VS, Schwartz CE, Smith RJ: Branchio-oto-renal syndrome: the mutation spectrum in EYA1 and its phenotypic consequences. Hum Mutat 2004, 23:582-589.

17. Orten DJ, Fischer SM, Sorensen JL, Radhakrishna U, Cremers CW, Marres HA, Van Camp G, Welch KO, Smith RJ, Kimberling WJ: Branchio-oto-renal syndrome (BOR): novel mutations in the EYA1 gene, and a review of the mutational genetics of BOR. Hum Mutat 2008, 29:537-544.

18. Abdelhak S, Kalatzis V, Heilig R, Compain S, Samson D, Vincent C, Levi-Acobas F, Cruaud C, Le Merrer M, Mathieu M, König R, Vigneron J, Weissenbach J, Petit C, Weil D: Clustering of mutations responsible for branchio-oto-renal (BOR) syndrome in the eyes absent homologous region (eyaHR) of EYA1. Hum Mol Genet 1997, 6:2247-2255. 
19. Vervoort VS, Smith RJ, O'Brien J, Schroer R, Abbott A, Stevenson RE, Schwartz CE: Genomic rearrangements of EYA1 account for a large fraction of families with BOR syndrome. Eur J Hum Genet 2002, 10:757-766.

20. Gu JZ, Wagner MJ, Haan EA, Wells DE: Detection of a megabase deletion in a patient with branchio-oto-renal syndrome (BOR) and tricho-rhinophalangeal syndrome (TRPS): implications for mapping and cloning the BOR gene. Genomics 1996, 31:201-206.

21. Krug P, Moriniere V, Marlin S, Koubi V, Gabriel HD, Colin E, Bonneau D, Salomon R, Antignac C, Heidet L: Mutation screening of the EYA1, SIX1, and SIX5 genes in a large cohort of patients harboring branchiooto-renal syndrome calls into question the pathogenic role of SIX5 mutations. Hum Mutat 2011, 32:183-190.

22. Sanchez-Valle A, Wang X, Potocki L, Xia Z, Kang SH, Carlin ME, Michel D, Williams P, Cabrera-Meza G, Brundage EK, Eifert AL, Stankiewicz P, Cheung SW, Lalani SR: HERV-mediated genomic rearrangement of EYA1 in an individual with branchio-oto-renal syndrome. Am J Med Genet A 2010, 152A:2854-2860

23. Paces J, Pavlicek A, Zika R, Kapitonov W, Jurka J, Paces V: HERVd: the Human Endogenous RetroViruses Database: update. Nucleic Acids Res 2004, 32:D50.

24. Jern P, Coffin JM: Effects of retroviruses on host genome function. Annu Rev Genet 2008, 42:709-732.

25. Salm MP, Horswell SD, Hutchison CE, Speedy HE, Yang X, Liang L, Schadt EE, Cookson WO, Wierzbicki AS, Naoumova RP, Shoulders CC: The origin, global distribution, and functional impact of the human 8 p23 inversion polymorphism. Genome Res 2012, 22:1144-1153.

26. Brophy PD, Alasti F, Darbro BW, Clarke J, Nishimura C, Cobb B, Smith RJ, Manak JR: Genome-wide copy number variation analysis of a Branchio-oto-renal syndrome cohort identifies a recombination hotspot and implicates new candidate genes. Hum Genet 2013, 132:1339-1350

27. Shuvarikov A, Campbell IM, Dittwald P, Neill NJ, Bialer MG, Moore C, Wheeler PG, Wallace SE, Hannibal MC, Murray MF, Giovanni MA, Terespolsky D, Sodhi S, Cassina M, Viskochil D, Moghaddam B, Herman K, Brown CW, Beck CR, Gambin A, Cheung SW, Patel A, Lamb AN, Shaffer LG, Ellison JW, Ravnan JB, Stankiewicz P, Rosenfeld JA: Recurrent HERV-H-Mediated 3q13.2-q13.31 Deletions Cause a Syndrome of Hypotonia and Motor, Language, and Cognitive Delays. Hum Mutat 2013, 34:1415-1423.

28. Sanggaard KM, Rendtorff ND, Kjaer KW, Eiberg H, Johnsen T, Gimsing S, Dyrmose J, Nielsen KO, Lage K, Tranebjaerg L: Branchio-oto-renal syndrome: detection of EYA1 and SIX1 mutations in five out of six Danish families by combining linkage, MLPA and sequencing analyses. Eur J Hum Genet 2007, 15:1121-1131.

29. Isidor B, Pichon O, Redon R, Day-Salvatore D, Hamel A, Siwicka KA Bitner-Glindzicz M, Heymann D, Kjellen L, Kraus C, Leroy JG, Mortier GR, Rauch A, Verloes A, David A, Le Caignec C: Mesomelia-synostoses syndrome results from deletion of SULF1 and SLCO5A1 genes at 8q13. Am J Hum Genet 2010, 87:95-100.

30. Verloes A, David A: Dominant mesomelic shortness of stature with acral synostoses, umbilical anomalies, and soft palate agenesis. Am J Med Genet 1995, 55:205-212.

31. Pfeiffer RA, Hirschfelder $H$, Rott HD: Specific acromesomelia with facial and renal anomalies: a new syndrome. Clin Dysmorphol 1995, 4:38-43.

32. Leroy JG, Claus L, Lee B, Mortier GR: Mesomelic dysplasia with specific autopodal synostoses: a third observation and further delineation of the multiple congenital anomaly syndrome. Pediatr Pathol Mol Med 2003, 22:23-35.

doi:10.1186/s12881-014-0090-9

Cite this article as: Chen et al:: Recurrent 8q13.2-13.3 microdeletions associated with Branchio-oto-renal syndrome are mediated by human endogenous retroviral (HERV) sequence blocks. BMC Medical Genetics 2014 15:90

\section{Submit your next manuscript to BioMed Central and take full advantage of:}

- Convenient online submission

- Thorough peer review

- No space constraints or color figure charges

- Immediate publication on acceptance

- Inclusion in PubMed, CAS, Scopus and Google Scholar

- Research which is freely available for redistribution

Submit your manuscript at www.biomedcentral.com/submit
Ciomed Central 